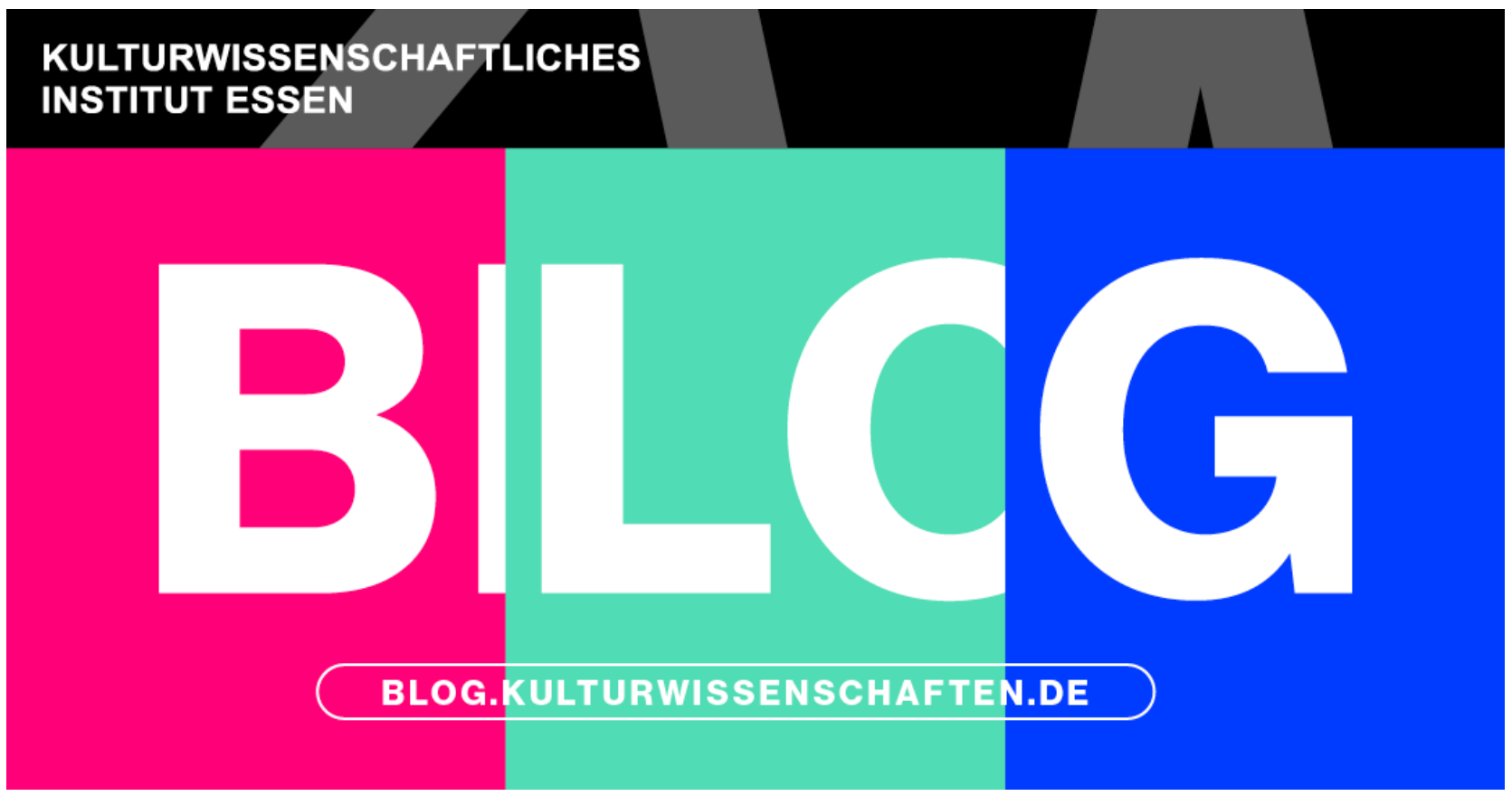

\title{
Die Popularisierung der professionellen Medizin
}

Erschienen in: Populäre Expertise | Popular Expertise

Von: Cornelius Schubert

[Dieser Beitrag in der Reihe „Populäre Expertise" erscheint parallel auf dem KWI-Blog. und dem Blog_des SFB 1472 „Transformationen des Populären“.]

Die Gegenüberstellung von populärer und professioneller Expertise ist ein wiederkehrendes Thema in der Entstehung der modernen Medizin. Entgegen den aktuellen Forderungen, durch Wissenschaftskommunikation stärker von der professionellen in die populäre Sphäre zu treten, lässt sich für die Entwicklung der modernen Medizin eher der umgekehrte Weg beobachten: Die verbreiteten Heilpraktiken der „popular medicine“ verloren im 19. Jahrhundert an Einfluss zugunsten der akademisch ausgebildeten Ärzteschaft. In weiten Teilen hat die professionelle Medizin die ehemals populären Heilkünste verdrängt, nur um selbst zum populären, d.h. in diesem Falle dominanten, Erklärungsrahmen für Gesundheit und Krankheit zu avancieren.

Die Popularisierung der (modernen, westlichen, Bio-) Medizin ist dabei das Produkt einer spezifischen Entwicklung, in der das Verhältnis von Expertïnnen und Laiïnnen neu verhandelt wird (nicht ohne zu vergessen, dass auch die Protagonisten dieser Medizin wie Louis Pasteur oder Robert Koch mit erheblichem Widerstand ihrer eigenen Kollegen zu kämpfen hatten). Sehr passend wird die Popularisierung der Medizin im englischen Titel von Bruno Latours Buch als „Pasteurisierung Frankreichs“ bezeichnet. ${ }^{1}$ Dort gilt es zunächst, Beachtung für die neue Medizin zu finden, ihren Deutungsanspruch zu belegen und durch Allianzen zu festigen - etwa durch ökonomische und politische Motive zur 
Erhaltung der Arbeits- und Kampfkraft in der Bevölkerung. Aus dieser Position werden nun die Erkenntnisse der Medizin in größerem Maßstab popularisiert, d.h. allgemein verständlich gemacht (etwa über Kriegsmetaphern in der Infektiologie) und gesellschaftlich implementiert (etwa über Hygienekonzepte).

Diese knappe Skizze soll als Kontrastfolie dienen, um die Gegenüberstellung von populärer und professioneller Expertise, wie sie oft in verkürzten Vorstellungen von Wissenschaftskommunikation zu finden ist, an einigen Punkten zu hinterfragen. Am historischen Beispiel der Medizin zeigt sich, wie die Auseinandersetzung zwischen professioneller und populärer Medizin mit der Unterscheidung von wahr und falsch aufgeladen wird. Während die universitäre Medizin einen übergreifenden Wahrheitsanspruch erhebt, gelten die anderen Heilkunden als tendenziell falsch und fehlerhaft, als Scharlatanerie und Quacksalbertum. Die epistemische Aufwertung der ersteren bei gleichzeitiger Abwertung der letzteren bestimmt somit auch das Verhältnis von professionell und populär als klar erkennbare high/low-Differenz. Im Zuge der Popularisierung der modernen Medizin wird die bis dahin populäre Medizin gewissermaßen „pasteurisiert“: Das biomedizinische Paradigma gewinnt gegenüber anderen Heilungskosmologien an Beachtung, ohne diese jedoch gänzlich verdrängen zu können. Zeitgleich stärkt dieser Prozess die Differenz zwischen Expertïn und Laiïn sowie die gehobene Stellung der Expertïnnen. Popularisierung und Professionalisierung sind in diesem Fall keine Gegensätze, sondern gehen Hand in Hand.

Die Wissenschaftskommunikation über die Medizin besteht in der zweiten Hälfte des 19. Jahrhunderts vornehmlich aus der Berichterstattung in populären Printmedien, zu der auch ab und zu die Verballhornung, Spott und Satire gehören. ${ }^{2}$ Insgesamt bildet sich aber ein gängiger Topos der Berichterstattung heraus: die sensationellen Entdeckungen der neuartigen Labormedizin durch heroische Forscherpersönlichkeiten wie Pasteur oder Koch. Die nachweislichen Erfolge der Mikrobiologie, die wissenschaftlichen Durchbrüche, die einer Vielzahl von Erkrankungen den Schrecken nahmen, erzeugten eine enge Koppelung zwischen der neuen laborbasierten Medizin und der Lebenswelt der Betroffenen, die sich über ein umfassendes Fortschrittsnarrativ gut in populäre Berichterstattung ummünzen ließ. Im 20. Jahrhundert kamen etwa Comics über die Entdeckungen der Medizin hinzu, teilweise gesponsert von Herstellerfirmen, sowie Anzeigekampagnen für Schutzimpfungen vor Diphterie oder Kinderlähmung. Die Popularisierung der Medizin wird demnach von mehreren Stellen vorangetrieben, nicht allein von der Medizin. Verlage, Hersteller, aber auch Gesundheitsbehörden sind ebenso beteiligt, vielleicht stärker als die universitäre Medizin selbst. Populäre und professionelle Expertise überlagern sich ein Stück weit durch die Popularisierung der modernen Medizin - auch wenn dies in der Regel dem paternalistischen Muster von Anweisung und Folgebereitschaft im asymmetrischen Expertïnnen-Laiïnnen-Verhältnis entspricht.

Eine breitere Kritik am paternalistischen Modell entwickelt sich in der zweiten Hälfte des 20. Jahrhunderts (Stichwort: shared decision-making) und auch die vermeintlichen Laiïnnen reklamieren zunehmend eigenständige Expertise für sich. Insbesondere die Selbsthilfegruppen chronisch Erkrankter verschaffen sich Gehör und im Zusammenhang mit politischem Aktivismus wird eine wirkungsvolle Gegenposition zu biomedizinischen 
Standardverfahren erkämpft, wie sich an der AIDS-Forschung der 1990er Jahr eindrücklich zeigen lässt. ${ }^{3}$ Das bedeutet aber nicht, dass diese Vorstöße sich allgemein gegen das biomedizinische Paradigma professioneller medizinischer Expertise wenden, vielmehr wollen sie deren Erkenntnisraum erweitern. Die AIDS-Aktivistïnnen, beispielsweise, eignen sich professionelles medizinisches Wissen an, um eine Veränderung der Zulassungskriterien für klinische Studien zu erreichen. Sie werden zu Laien-Expertïnnen, die durchaus an der Entwicklung der AIDS-Forschung Anteil haben, ohne aber einschlägig medizinisch ausgebildet zu sein. Diese Durchlässigkeit an den Grenzen der Wissenschaft lässt sich nicht nur im medizinischen Kontext beobachten. Schon seit den 1970er Jahren wurden die Forderungen nach einer Demokratisierung bzw. zivilgesellschaftlichen Mitsprache an wissenschaftlich-technischen Großprojekten lauter. Wissenschaftliche Expertise musste sich erklären und legitimieren, wenn es um zentrale gesellschaftliche Fragen ging. Und sie war mit lebensweltlicher Erfahrung konfrontiert, die ebenso Deutungshoheit beanspruchte. In der Folge rückte die Wissenschaft in vielfältigen Partizipationsformaten näher an die Bürgerïnnen heran und für die Wissenschaftskommunikation entwickelten sich in diesen Arenen und Foren neue Formen des Austausches.

Auch diese Entwicklung zeigt, dass die mittlerweile populär gewordene Expertise der Medizin sich tendenziell weiter in Transformation befindet. Ein nächster Schub wurde mit der Verbreitung des Internets eingeläutet. Die Verfügbarkeitsschwelle von Fachinformationen sank und die Vernetzungsmöglichkeiten für Patientïnnen wurden vielfältiger. Schon bald nach der Jahrtausendwende wurde vermehrt von „informierten“ Patientïnnen gesprochen. Einerseits waren damit Patientïnnen gemeint, die durch Ärztïnnen in besonderer Weise umfassend und mündig aufgeklärt wurden; andererseits ging es um Patientïnnen, die sich selbständig - meist auf Webseiten oder Internetforen informierten und ihre Ärztïnnen in der nächsten Sprechstunde mit den erworbenen Erkenntnissen konfrontieren. ${ }^{4}$ Die Gatekeeperfunktion der ärztlichen Profession, die den Zugang zu professionellem Wissen und Gesundheitsdienstleistungen regelte, geriet unter Druck. Zumindest musste in Arztpraxen einiges an Interaktionsarbeit geleistet werden, um die professionelle Expertise von der frei verfügbaren Information zu trennen. Bei diesen frei verfügbaren Informationen handelte es sich nicht selten aber um medizinische Fachbeiträge. Die Emanzipation und Ermündigung von Patientïnnen bedeutet in diesen Fällen keine Entmachtung des biomedizinischen Paradigmas, sondern seine Verbreitung und Aneignung, die wiederum auf die Medizin zurückwirkt. Was von Patientïnnen auf diese Weise erzeugt wird, unterscheidet sich als populäre Expertise von der popularisierten Expertise der professionellen Medizin, widerspricht ihr aber nicht unbedingt. Populäre Internetforen wie patientslikeme.com stehen nicht in Opposition zur professionellen Medizin, sie versuchen vielmehr durch die Beteiligung von Patientïnnen relevante Daten für den Umgang mit Krankheiten zu generieren.

Ein anderes Beispiel ist die öffentliche Aushandlung dessen, was als professionelle medizinische Expertise gilt. Unter dem Hashtag \#Globukalypse wird auf Twitter gegen die Erstattung von homöopathischen Mitteln durch die Krankenkassen argumentiert. Dort finden sich zwar viele Medizinerïnnen, aber darüber hinaus auch etliche Unterstützerïnnen ohne medizinische Ausbildung, die teils vehement auf Seiten der 
evidenzbasierten Medizin streiten. In dieser Kontroverse verläuft die Konfliktlinie nicht so sehr zwischen der professionellen Medizin auf der einen und einer populären Meinung auf der anderen Seite, sondern zwischen Lagern beider Seiten, die jeweils unterschiedliche Wissensbestände und Evidenzpraktiken für sich reklamieren. Das Ringen um die Anerkennung der Homöopathie auf Plattformen wie Twitter lässt sich daher nicht einfach auf die Dichotomie professionell vs. populär und damit die Unterscheidung wahr vs. falsch reduzieren. Im Sinne der Wissenschaftskommunikation erlangen homöopathiekritische Ärzte wie Christian Lübbers (@drluebbers) mit aktuell knapp 68.000 Followerïnnen auf Twitter Popularität. Es mag eine Besonderheit der Wissenschaftskommunikation in der Medizin sein, dass sie ohnehin ständig in den Sprechstunden von Ärztïnnen geleistet wird und dass das Verhältnis von Expertïnnen und Laiïnnen in der Medizin weitgehend durch das Verhältnis der Profession zu ihrer Klientel institutionalisiert ist. Die allgemeine Präsenz von medizinischen Themen in der medialen Berichterstattung (im Gegensatz zu anderen Disziplinen) unterstreicht diese Ausnahmestellung und wird durch die Pandemie erneut verstärkt.

Ich möchte noch auf eine neuere Entwicklung zu sprechen kommen, die die Verhältnisse von populärer und professioneller Expertise unter Umständen weiter transformiert. Das betrifft die Popularisierung biomedizinischer Messverfahren, insbesondere über Aktivitätstracker oder Smartwatches sowie die dazugehörigen Apps. War die Erzeugung digitaler Körperdaten, wie etwa Puls- oder Sauerstoffmessung, lange Zeit eine Domäne der professionellen Medizin, so sind diese Messverfahren mittlerweile Teil des Lifestyles. Einzelne Communities, wie die des „Quantified Self“, betreiben eine genaue Aufzeichnung von Körperdaten und etablieren dadurch eigene Evidenzpraktiken, die sich jedoch stark am Vorbild naturwissenschaftlicher Erkenntnis orientieren. Die Popularisierung biomedizinscher Kennzahlen, ihre verbreitete Messung mittels mobiler Sensoren in Alltag und Freizeit, fordert gleichzeitig zur intensiveren Beschäftigung mit ihnen auf. Auch in diesem Fall kann nicht von einer schlichten Ausdehnung der medizinischen Sphäre und einer weiteren Popularisierung professioneller Expertise ausgegangen werden. Es ergeben sich durch die Devices und Apps jedoch neue Kommunikationsachsen, die sowohl von Seite der Ärztïnnen sowie der Patientiïnnen noch ausgelotet werden müssen. ${ }^{5}$ Inwieweit digitale Gesundheitsanwendungen (DiGA) zu weiteren Grenzverschiebungen im Verhältnis von Expertïnnen und Laiïnnen führen, bleibt eine offene Frage. Es ist aber anzunehmen, dass sich grundlegende Änderungen nicht sprunghaft vollziehen werden, denn auch in sehr aktiven Communities wie bei Typ I Diabetes bleibt die professionelle medizinische Expertise eine zentrale Größe.

Das Beispiel der Medizin zeigt, dass sich unterschiedliche Gemengelagen zwischen populären und professionellen Expertisen finden lassen. Die Popularisierung professionellen Wissens als populäre Expertise ist weiterhin - und in Pandemiezeiten umso mehr - gefragt. Zugleich zeigt sich, dass die professionelle Autorität nicht unwidersprochen bleibt. Die gesteigerte Verfügbarkeit von medizinischen Informationen und digitalen Körperdaten ermöglichen es Laiïnnen, eigene Expertise aufzubauen und auf dieser Basis neu mit Expertïnnen ins Gespräch zu kommen. Daher lohnt es sich, differenziert auf die Kommunikationsprozesse zwischen Wissenschaft und Gesellschaft 
bzw. zwischen Expertïnnen und Laiïnnen zu schauen. Die Verschiebungen in Fragen der Expertise stellen das dominante Modell nicht grundsätzlich in Frage, aber die NeuJustierungen lassen erkennen, dass Transformationsprozesse am Werk sind.

\section{References}

1. Latour, Bruno (1988): The Pasteurization of France. Cambridge: Harvard University Press.

2. Hansen, Bert (2009): Picturing Medical Progress from Pasteur to Polio. A History of Mass Media Images and Popular Attitudes in America. New Brunswick: Rutgers University Press.

3. Epstein, Steven (1998): Impure Science. AIDS, Activism, and the Politics of Knowledge. Berkeley: University of California Press.

4. Baumgart, Julia (2010): Ärzte und informierte Patienten: Ambivalentes Verhältnis. In: Deutsches Ärzteblatt 107 (51-52): A 2554-6.

5. Radić, Marija; Donner, Isabelle; Waack, Matthias; Brinkmann, Carolin; Stein, Leon; Radić, Dubravko (2021): Digitale Gesundheitsanwendungen. Die Akzeptanz steigern. In: Deutsches Ärzteblatt 118 (6), A 286-92.

SUGGESTED CITATION: Schubert, Cornelius: Die Popularisierung der professionellen Medizin, in: KWI-BLOG, [https://blog.kulturwissenschaften.de/die-popularisierung-derprofessionellen-medizin/], 07.02.2022

DOI: https://doi.org/10.37189/kwi-blog/20220207-0830 
DuEPublico

Duisburg-Essen Publications online
DE SISEB R R G

offen im Denken

$\mathbf{U b} \mid \begin{aligned} & \text { universitäts } \\ & \text { bibliothek }\end{aligned}$

Dieser Text wird via DuEPublico, dem Dokumenten- und Publikationsserver der Universität Duisburg-Essen, zur Verfügung gestellt. Die hier veröffentlichte Version der E-Publikation kann von einer eventuell ebenfalls veröffentlichten Verlagsversion abweichen.

DOI: $\quad 10.37189 / \mathrm{kwi}-\mathrm{blog} / 20220207-0830$

URN: urn:nbn:de:hbz:464-20220207-102950-6 